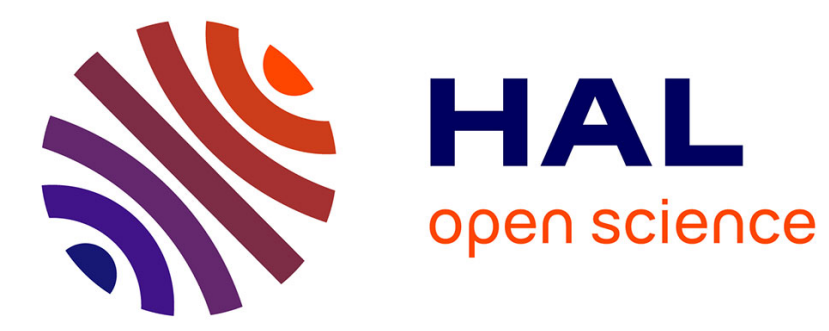

\title{
Mycobacterial lipolytic enzymes: A gold mine for tuberculosis research
}

Luc Dedieu, C. Serveau-Avesque, L. Kremer, Stéphane Canaan

\section{To cite this version:}

Luc Dedieu, C. Serveau-Avesque, L. Kremer, Stéphane Canaan. Mycobacterial lipolytic enzymes: A gold mine for tuberculosis research. Biochimie, 2013, 95 (1), pp.66-73. 10.1016/j.biochi.2012.07.008 . hal-02646757

\section{HAL Id: hal-02646757 \\ https://hal.inrae.fr/hal-02646757}

Submitted on 29 May 2020

HAL is a multi-disciplinary open access archive for the deposit and dissemination of scientific research documents, whether they are published or not. The documents may come from teaching and research institutions in France or abroad, or from public or private research centers.
L'archive ouverte pluridisciplinaire HAL, est destinée au dépôt et à la diffusion de documents scientifiques de niveau recherche, publiés ou non, émanant des établissements d'enseignement et de recherche français ou étrangers, des laboratoires publics ou privés.

\section{(ㅇ)(1) $\$$}

Distributed under a Creative Commons Attribution - NonCommercial - NoDerivatives| 4.0 
Review

\title{
Mycobacterial lipolytic enzymes: A gold mine for tuberculosis research
}

\author{
L. Dedieu ${ }^{a}$, C. Serveau-Avesque ${ }^{\text {a }}$, L. Kremer ${ }^{\text {b,c }}$, S. Canaan ${ }^{\text {a,* }}$ \\ ${ }^{a}$ CNRS UMR7282-Aix-Marseille Université - Enzymologie Interfaciale et Physiologie de la Lipolyse, SDV, 31 chemin Joseph Aiguier, 13402 Marseille Cedex 20, France \\ ${ }^{\mathrm{b}}$ Laboratoire de Dynamique des Interactions Membranaires Normales et Pathologiques, Universités de Montpellier 2 et 1, CNRS UMR 5235, case 107, Place Eugène Bataillon, \\ 34095 Montpellier Cedex 05, France \\ ${ }^{\mathrm{C}}$ INSERM, DIMNP, Place Eugène Bataillon, 34095 Montpellier Cedex 05, France
}

Keywords:

Localization

Cell wall

Physiopathology

Immunogenicity

Diagnosis

Mycobacterium

\begin{abstract}
A B S T R A C T
Tuberculosis (TB) is one of the deadliest infectious diseases worldwide with a strong impact in developing countries. Mycobacterium tuberculosis, the etiological agent of TB, has a high capacity to evade the host immune system and establish a chronic, asymptomatic and latent infection. In a latent TB infection, persistent bacilli are present in a non-replicating dormant state within host granulomas. During reactivation, bacilli start replicating again leading to an active TB infection that can be highly contagious. Mycobacterial lipids and lipolytic enzymes are thought to play important physiological roles during dormancy and reactivation. The role of lipolytic enzymes in the physiology of M. tuberculosis and physiopathology of the disease will be discussed in this review, with an emphasis on the secreted or cell wall-associated, surface exposed lipolytic enzymes characterized to date. Studies on the localization, enzymatic activity and immunological properties of these enzymes highlighted their possible usefulness as new diagnostic markers in the fight against TB.
\end{abstract}

\section{Introduction}

With 8.8 million new infections and 1.5 million deaths in 2010 [1], tuberculosis (TB) continues to be one of the most threatening and deadly diseases in the world. The lack of reliable control measures, emergence of multi-drug resistant strains of the causative bacterium, Mycobacterium tuberculosis, and the ability of the latter to remain undetected in a dormant stage in infected individuals, are some of the principal reasons for the global TB epidemic. Therefore, there is an urgent need for alternative methods of diagnosis, vaccination, and treatment. In this context, enzymes involved in mycobacterial lipid metabolism have generated considerable interest because of the increasing evidence of their role in the physiopathology of the disease.

Indeed, a large number of chemically complex lipids with unique characteristics are associated with the cell envelope of M. tuberculosis, which distinguishes it from other bacteria [2]. Despite numerous studies on the chemistry, biological functions, and biosynthetic pathways of the cell wall lipids, the intricate architecture of the envelope remains enigmatic. Characteristic

Abbreviations: TAG, triacylglycerol; FM, foamy macrophage; LB, lipid body; ILI intracellular lipidic inclusion; TB, tuberculosis; PPD, protein purified derivative.

* Corresponding author. Tel.: +33 4911640 93; fax: +33 491715857.

E-mail address: canaan@imm.cnrs.fr (S. Canaan). mycobacterial (glyco)lipids including mycolic acids, phosphatidyl inositol mannosides, phthiocerol dimycocerosates, as well as lipoglycans such as lipomannan and lipoarabinomannan, that have been the focus of extensive research are considered as major virulence determinants in mycobacteria [3].

In contrast to the cell wall-associated lipids, intracytoplasmic lipids have been largely neglected. It is only in the last decade that the link between intracellular lipids and physiopathology of the disease has been investigated. It was shown that during infection, M. tuberculosis accumulates intracellular lipid inclusions (ILI) [4] that are probably derived from lipids of the host cell membrane [5-8]. By this way, M. tuberculosis stores fatty acids in the form of triacylglycerol (TAG) [9-12] for use as a source of carbon and energy during dormancy. In this non-replicating stage, bacilli could persist for several decades and are particularly recalcitrant to treatment. In reactivation cases, TAGs are used to fuel the resumption of cellular replication causing an active infection [13]. However, this reactivation of latent infection usually occurs more frequently in immunocompromised individuals.

Accumulation of lipids derived from host cells and their consumption have been demonstrated in several bacteria [14], and in mycobacterial species such as $M$. tuberculosis [4,9,12,15-17], Mycobacterium bovis BCG [13,18], Mycobacterium leprae [19] and Mycobacterium smegmatis [4,10].

Typically, the tubercle bacilli enter the body by inhalation of aerosols, and reach the lungs where they are phagocytosed by 
Version définitive du manuscrit publiée dans / Final version of the manuscript published in :

Biochimie (2013), Vol. 95, p. 66-73, DOI: 10.1016/j.biochi.2012.07.008

Journal homepage : www.elsevier.com/locate/biochi

pulmonary alveolar macrophages. The subsequent host response consists of recruitment of macrophages, lymphocytes and dendritic cells, leading to the formation of a highly organized cellular structure, termed granuloma, a major histopathological hallmark of TB. In this structure, macrophages containing bacilli accumulate intracytoplasmic lipid bodies (LB), mainly composed of neutral lipids surrounded by a phospholipid layer, that gives the macrophages their characteristic foamy appearance (Fig. 1A) [12]. Within the foamy macrophages (FM), phagocytosed bacteria preferentially metabolize lipids rather than carbohydrates [20], a view that is supported by evidence of up-regulation of several mycobacterial genes involved in lipid metabolism [17]. At this stage, the intraphagosomal bacteria acquire and accumulate ILI in their cytoplasm (Fig. 1B and C) and persist in a non-replicating state, ultimately leading to dormancy, i.e., a latent infection. As demonstrated in an in vitro model of human granulomas [12], these lipid droplets (LB and ILI) serve as sources of carbon and energy for the dormant bacilli within granulomas, thereby aiding reactivation that can lead to an active TB infection [21]. In addition to FM, ILI accumulation has also been reported in $M$. tuberculosis-infected adipocytes [7] and in M. leprae-infected macrophages and Schwann cells [22].

Biochemical analyses have revealed that $M$. tuberculosis ILIs are mainly composed of TAGs. The latter are derived from free fatty acids that may be imported from the host or synthesized de novo [9]. TAGs accumulate during mycobacterial growth and the amount of intracellular TAGs peaks in the late exponential growth phase [23] and the non-replicating phase [15]. Further, it has been shown that expression of $M$. tuberculosis-specific lipase genes is strongly elevated during dormancy [16], and that in the reactivated bacilli, a reduction in TAG levels coincides with an increase in TAG lipase activity [13]. Thus, enzymes involved in lipid degradation appear to serve important physiological functions and contribute to the extraordinary capacity of survival of $M$. tuberculosis within the infected host.

Despite lipolytic activities have been described in mycobacteria many years ago, the associated enzymes, lipases and phospholipases, have only been the focus of intense research for the last few years [24-27]. Lipases and phospholipases are peculiar molecules that provide a metabolic turnover of lipids and can be defined as essential biocatalysts for the hydrolysis of esters containing longchain fatty acids. Lipases have been well characterized in the animal kingdom [28-30], particularly in mammals where they play important roles in fat digestion, lipoprotein metabolism, as well as in mobilization of fat stored in adipocytes. Because of their fundamental role in lipid metabolism, lipolytic enzymes have received attention in human health as therapeutic agents against obesity, cardiovascular diseases or as adjuvants in treatment of chronic pancreatitis or in cystic fibrosis. In case of mycobacterial lipases, an interesting aspect that has recently come to light, is their immunogenicity, which has prompted their use as biomarkers of active TB [31,32].

In this review, we will discuss the recent advances in understanding of the biological properties of mycobacterial lipolytic enzymes, which have opened up exciting possibilities of their

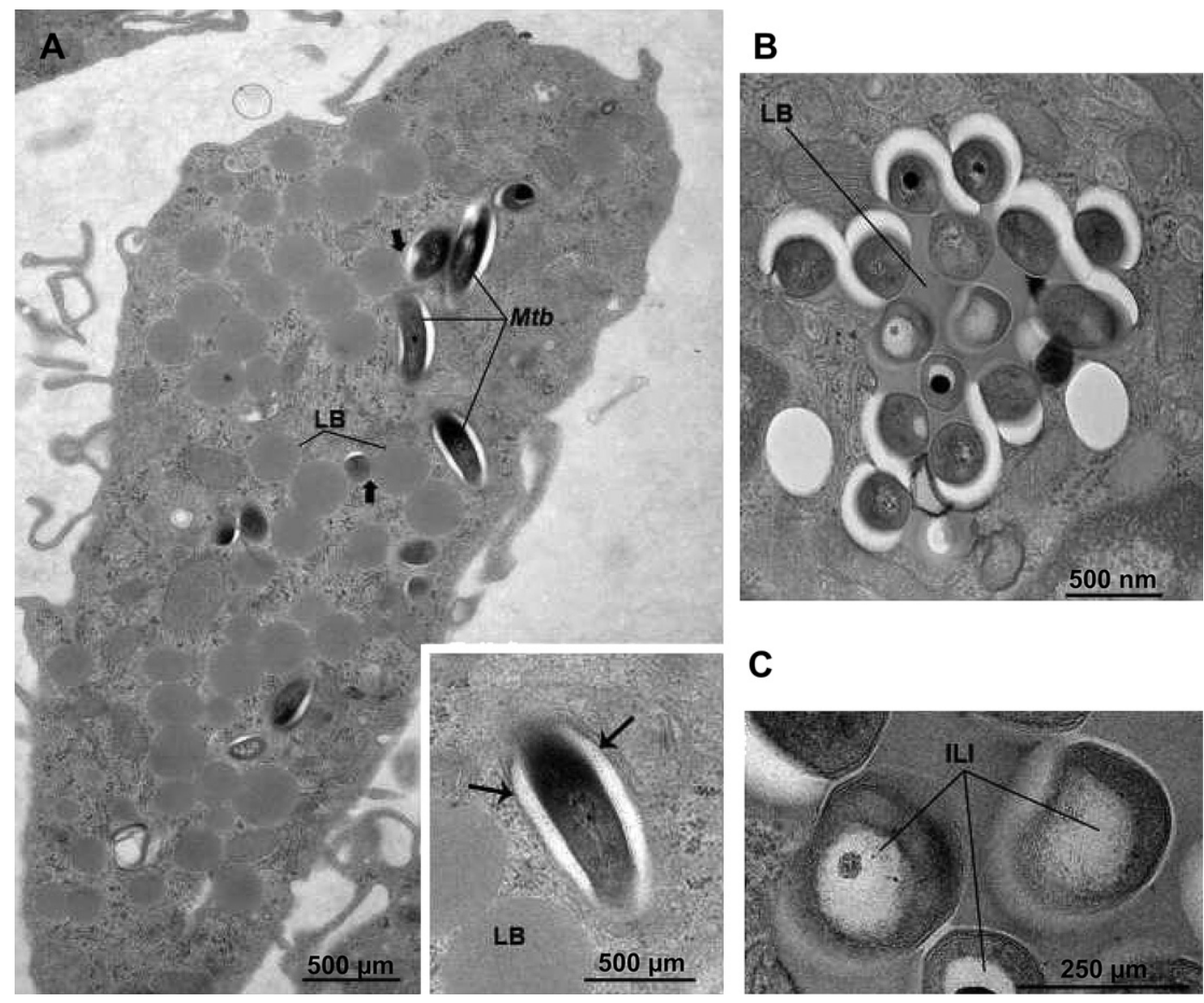

Fig. 1. Intracellular lipid bodies (LB) and phagosomes in M. tuberculosis-infected foamy macrophages. Granulomas were obtained in vitro by infection of peripheral blood mononuclear cells with $M$. tuberculosis H37Rv, and processed for electron microscopy at day 11 post-infection. (A) A typical foamy macrophage carrying LBs and phagosomes containing M. tuberculosis. Inset: arrows indicate the phagosomal membrane around the bacterium. (B) LB surrounded by several M. tuberculosis bacilli. Some of the bacteria display intracytoplasmic lipid inclusions (ILIs). (C) Enlarged view of (B) showing M. tuberculosis bacilli containing large ILIs (Adapted from Ref. [12]). 
applications in more accurate methods of diagnosis of active TB and as putative therapeutic targets.

\section{In silico identification of lipolytic enzymes in M. tuberculosis}

The analysis of $M$. tuberculosis genome revealed that this bacterium possesses an unusually large number of genes encoding putative enzymes involved in lipid metabolism [33]. Indeed, M. tuberculosis genome contains 250 genes encoding putative enzymes involved in the synthesis or degradation of lipids compared to 50 genes in Escherichia coli, which has a similar genome size. This feature, combined with the extremely high amount of lipids, representing $30-40 \%$ of the dry weight of $M$. tuberculosis, suggests that lipids and lipolytic enzymes play an important role in the mycobacterial life cycle and perhaps in virulence. In silico analyses identified the presence of around 30 putative genes encoding lipolytic enzymes (enzymes involved in lipids degradation), including 24 lipid/ester hydrolases, belonging to the so-called "Lip" family (LipC to LipZ). These have been annotated as putative esterases or lipases based on the presence of the consensus sequence GXSXG, which is a characteristic feature of members of the $\alpha / \beta$ hydrolase-fold family [34]. Based on their primary sequences, 28 of the $31 \mathrm{M}$. tuberculosis lipolytic enzymes are classified into different families: 1 belonging to the Candida parapsilosis lipase family, 1 to the human Bile Salt-Stimulated Lipase (BSSL) family, 12 to the human Hormone-Sensitive Lipase (HSL) family, 7 to the Fusarium solani cutinase (Cut) family, 3 to the monoglyceride lipase family, 3 not belonging to the above mentioned lipase family and 4 to the phospholipases C (PLC) family. Except for the PLCs, all other enzymes possess an active site comprising the characteristic catalytic triad Ser-His-Glu/Asp. However, biochemical characterization of these enzymes using specific substrates is a prerequisite to establish their true activities and physiological functions.

\section{Lipolytic enzymes in the mycobacterial cell wall architecture}

Immunolocalization studies have revealed the association of several M. tuberculosis lipolytic enzymes with the cell wall, thus raising the possibility of these proteins as structural components of the membrane and/or their potential role in enzymatic degradation of host cell lipids. Surface-exposed localization also suggests that they may participate in modulation of the immune response $[35,36]$ and/or in invasion of host cells $[5,8]$. This chapter will focus on the three enzymes discovered up to date, involved in the architecture of the cell wall and able to trigger a specific humoral response during infection.

\subsection{LipY: dual intra and extracellular localization and hydrolytic activity}

The primary sequence of LipY (Rv3097c) clearly identified this protein as a member of the HSL family, while the presence of an additional $\mathrm{N}$-terminal $\mathrm{PE}$ domain also qualified it as a member of the PE family of protein [16]. Biochemical characterization demonstrated that LipY is able to hydrolyze ILI-containing TAGs [16,35]. In M. tuberculosis under nutrient-deprived conditions, lipY expression was highly induced, accompanied by efficient utilization of accumulated TAGs in the absence of another source of carbon. In a lipY-deleted mutant of $M$. tuberculosis, TAG hydrolysis was severely impeded in vitro (Fig. 2A). From these results, it can be inferred that LipY is responsible for the utilization of TAGs

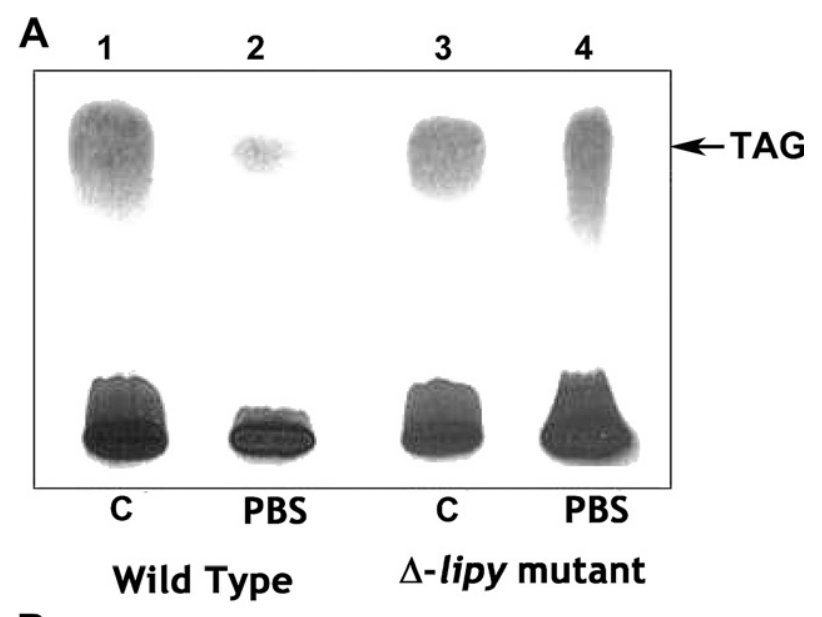

B

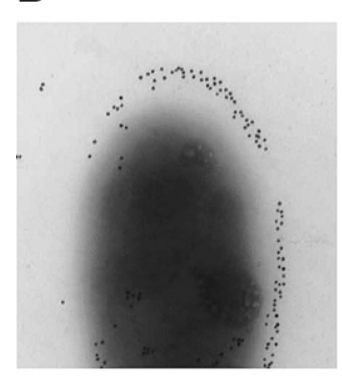

Wild Type

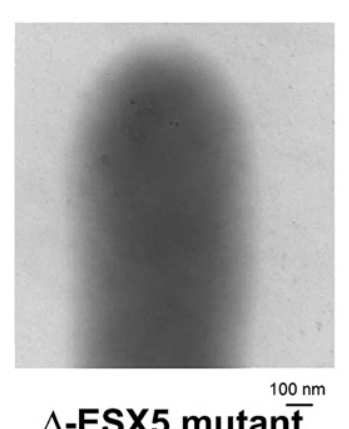

$\Delta$-ESX5 mutant
Fig. 2. Physiological function and dual localization of LipY. (A) LipY is a TAG hydrolase. Thin layer chromatography of lipids isolated from hypoxic cultures of M. tuberculosis wild type (WT) strain or LipY-deficient $(\Delta$-lipY) mutant strain, processed for visualization of TAGs. Levels of intracellularly accumulated TAGs (indicated by arrow) were similar in the two strains (lanes 1 and 3; controls, C); when cells were nutrientdeprived in phosphate-buffered saline (PBS) for $6 \mathrm{~h}$ (lanes 2 and 4), the WT strain utilized TAGs far more efficiently than the LipY-deficient mutant (Adapted from Ref. [16]). (B) LipY is exposed on mycobacterial surface. Electron microscopy showing immunogold localization of LipY on the cell surface in a M. marinum WT strain (left panel) but not in a M. marinum ESX-5 mutant strain (type 7 secretion system) (right panel) (Adapted from Ref. [37]).

accumulated in the bacterial cytoplasm during dormancy and/or during exit from dormancy, leading to the reactivation.

However, the activity of LipY on ILIs has been difficult to reconcile with its localization on the bacterial surface as demonstrated by immunogold electron microscopy [35]. Recent work by Daleke et al. [37] demonstrated that in Mycobacterium marinum, LipY was secreted extracellularly, which agreed with the presence of a PPE domain (similar to PE domain in M. tuberculosis) and the activity of the ESX-5 secretion pathway in this species (Fig. 2B). Recently, this ESX-5 has also been identified as the system for secretion of proteins carrying PE/PPE_PGRS domains in $M$. tuberculosis [38]. Interestingly, Zeng et al. [39] identified intracellular proteins capable of physical interactions with LipY, which may explain the retention of the lipase in the cytoplasm of the bacteria. These results suggest a dual cellular localization of LipY, intracytoplasmic or cell surface-associated following the cleavage of the PE/PPE N-terminal domain via ESX-5 machinery. Both localizations are in agreement with the action of LipY in the degradation of TAGs within ILIs and the hydrolysis of lipids from host LBs, respectively. Importantly, the observation of surface exposure of LipY led to the hypothesis that it may be accessible to the host immune system and may trigger a specific humoral response. This hypothesis has been confirmed by the detection of a robust anti-LipY antibody response in TB patients [35]. 
Collectively, these results validate the central role of LipY in TAG hydrolysis. Because LipY emerges as the major long-chain TAG hydrolase involved in TAG hydrolysis during nutrient starvation, one may postulate that LipY plays a key role in the acquisition of host lipids, by degrading TAGs present in the LBs in the FM.

\subsection{LipC: an immunogenic cell surface esterase}

Many antigenic proteins expressed during the early stages of active TB play important roles in host-pathogen interactions. Shen et al. [32] reported the strong immunogenicity of LipC, another member of the Lip family [33] belonging to the HSL family. Using an epitope mapping strategy, 6 immunodominant epitopes were identified, 5 of which mapped to residues exposed at the surface of the protein. Although predicted to be a cytoplasmic enzyme, it was demonstrated that LipC is a cell surface protein present in both the cell wall and the capsule of M. tuberculosis (Fig. 3A). In an effort to understand its biological relevance, recombinant LipC was purified and characterized biochemically, revealing an esterase activity on short-chain esters (Fig. 3B). A M. tuberculosis LipC-deleted strain was not affected in its growth rate or viability in vitro, and represents a useful tool to investigate whether LipC hydrolyzes fatty acyl chains on ester substrates from the host. In addition, LipC was capable of stimulating pro-inflammatory cytokines and chemokines in macrophages and pulmonary epithelial cells [32]. Taken together, these studies demonstrate that LipC is a novel, cell surface-associated esterase of $M$. tuberculosis that is highly immunogenic and possibly involved in the modulation of the host immune response.

\subsection{Rv0183: a monoglyceride lipase involved in degradation of host cell lipids}

Rv0183 shares 34\% and 36\% amino acid sequence identity with the monoglyceride lipases from rat and human, respectively, and represents the only monoglyceride lipase identified in mycobacteria [6]. Immunolocalization studies revealed the presence of
Rv0183 in the cell wall, leading to the hypothesis that it is likely involved in hydrolysis of host cell lipids. From an enzymatic point of view, Rv0183 exhibits a strong substrate preference for monoacylglycerols compared to diacylglycerols or TAGs, raising the possibility that it provides free fatty acids to mycobacteria. To address the in vivo role of this enzyme, a disrupted mutant of its ortholog in M. smegmatis, MSMEG_0220, was generated (Fig. 4A) [40]. This mutant displayed an unexpected colony morphotype compared to the parental strain (Fig. 4C-F). Its liquid culture was found to be more homogenous with less clumping of cells, presumably due to an alteration in the intercellular interactions (Fig. 4G-J). Both phenotypes were partially restored by complementation with a gene encoding either an active or an inactive MSMEG_0220 enzyme. Moreover, growth studies performed in medium supplemented with monoolein (MO) indicated that growth of the MSMEG_0220-disrupted mutant was impaired (Fig. 4B). Notably, Rv0183 could be detected in the serum of infected patients [31]. Moreover, heterologous expression of Rv0183 in mouse macrophages was found to induce cell apoptosis and to trigger expression of inflammatory factors such as IL-6, NF- $\kappa B$, TLR2 , TLR-6, TNF- $\alpha$ [41]. These results imply that Rv0183 is a structural component of the mycobacterial cell wall, hydrolyzes exogenous lipids and may strongly induce the immune response of the host.

\section{Cutinases: broad-range activity and cytotoxicity}

Cutinases are extracellular serine esterases able to degrade a large panel of substrates such as cutin, which is a polyester protecting plant leaves but also TAGs and phopholipids. Even if cutinases are mainly found in phytopathogenic organisms, seven genes related to the cutinase family have been found in the M. tuberculosis genome [33,42]. Among these 7 putative cutinase-like proteins, two secreted enzymes, Cfp21 (Rv1984c) and Cut4 (Rv3452) appear structurally related, sharing 52\% identity and 66\% homology. However, biochemical characterization showed that these enzymes exhibit marked differences with respect to their substrate and enzymatic specificities. While Cut4 behaves similar to a typical
A
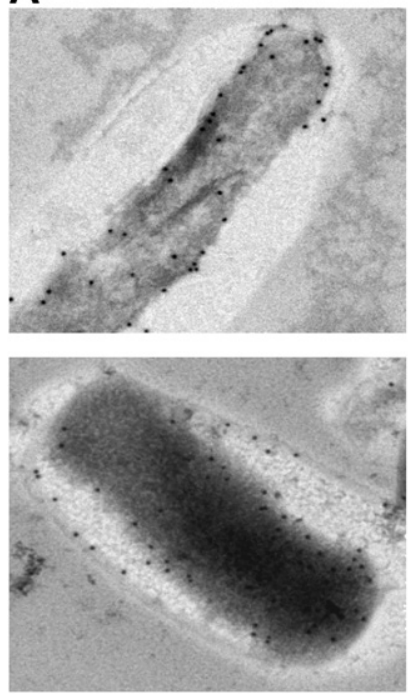

B

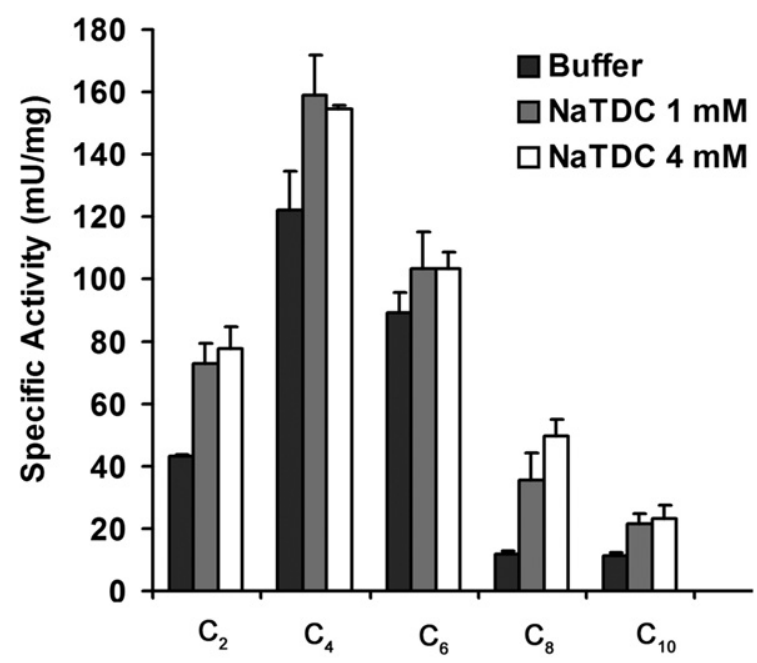

Fig. 3. Localization and enzymatic activity of LipC. (A) Mycobacterial cell surface localization of LipC. Immunoelectron microscopy of ultrathin sections of M. tuberculosis H37Rv (upper panel) and M. tuberculosis CDC1551 (lower panel) from in vitro cultures, probed with rabbit anti-LipC antibodies. (B) LipC has preference for short-chain esters. Enzymatic activity of recombinant LipC was measured using 8-p-nitrophenyl (PNP) esters with carbon chain lengths ranging from $C_{2}$ to $C_{10}$ as substrates. These PNP esters were mixed in a buffer containing 0,1 or $4 \mathrm{mM}$ sodium taurodeoxycholate (NaTDC). NaTDC is a bile salt used to solubilize insoluble lipids bearing fatty acid with long carbon chain. The activity on substrates of carbon lengths $\geq 12$ was undetectable. The mean values of specific activity corresponding to $1 \mu$ mol PNP released per min and per mg protein, are plotted (Adapted from Ref. [32]). 
Version définitive du manuscrit publiée dans / Final version of the manuscript published in :

Biochimie (2013), Vol. 95, p. 66-73, DOI: 10.1016/j.biochi.2012.07.008

Journal homepage : www.elsevier.com/locate/biochi
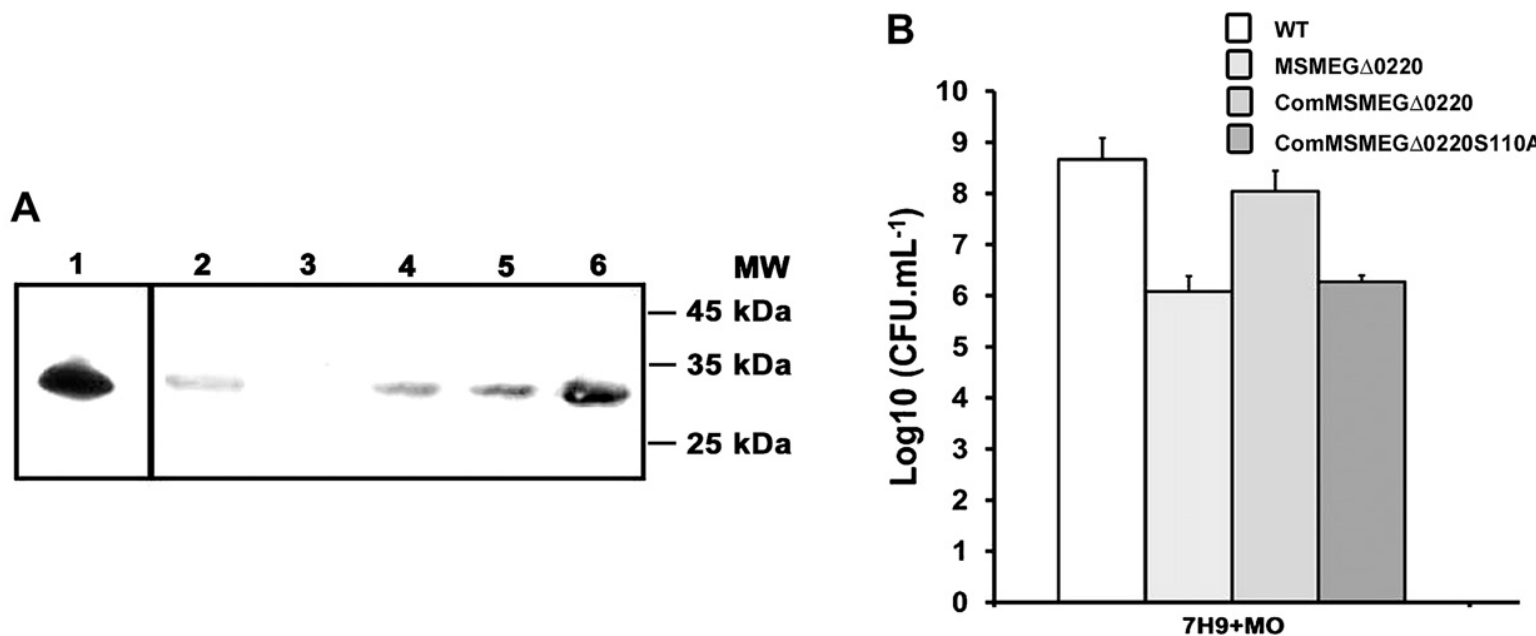

WT

C

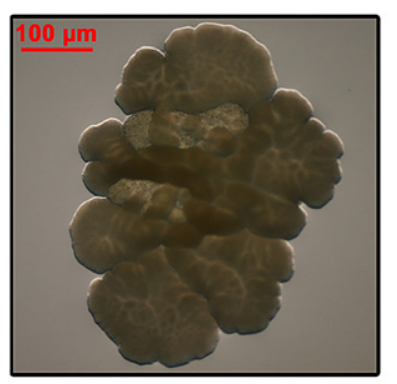

G

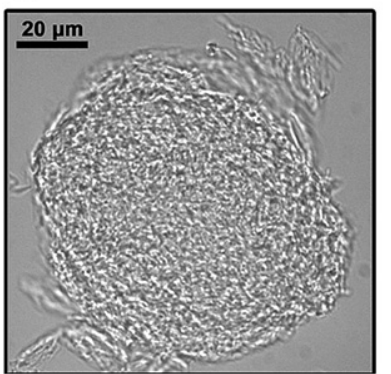

MSMEG $\triangle 0220$

D

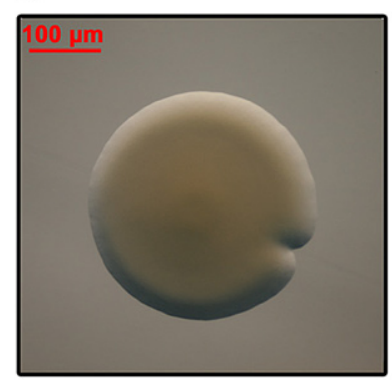

$\mathrm{H}$

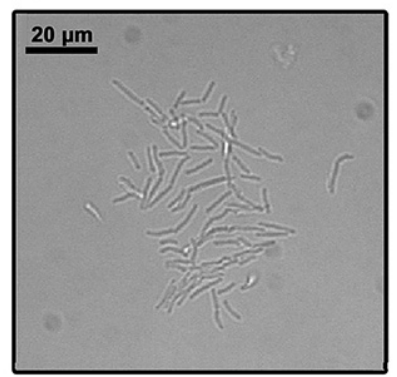

ComMSMEG $\triangle 0220$

E

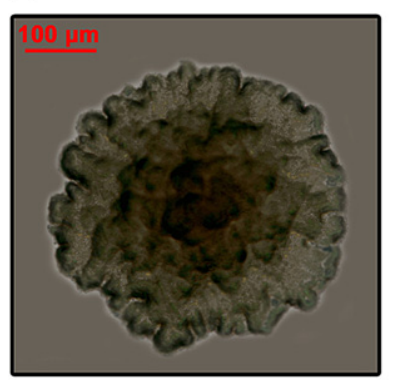

I

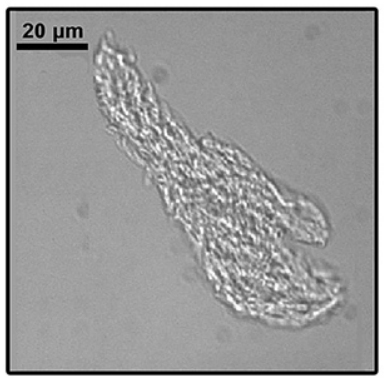

ComMSMEG $\triangle 0220$ S110A

$\mathbf{F}$

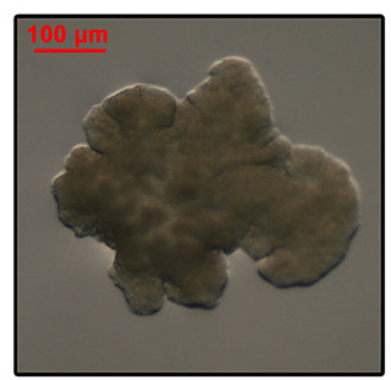

J

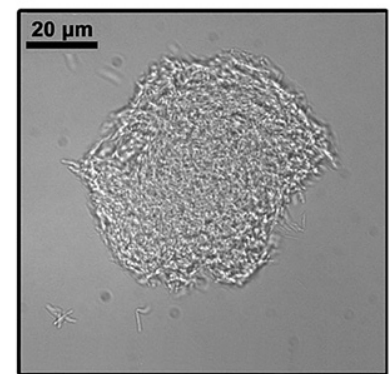

Fig. 4. Localization and physiological function of monoglyceride lipase, MSMEG_0220 of M. smegmatis and its homolog Rv0183 from M. tuberculosis. (A) MSMEG_0220 produced in M. smegmatis $\mathrm{mc}^{2} 155$. MSMEG_0220 protein in cell wall fractions from different strains was visualized by immunoblotting using purified polyclonal antibodies against recombinant Rv0183. Total protein $(120 \mu \mathrm{g})$ from cell wall fractions and purified recombinant proteins (100 ng) were loaded as follows: 1, recombinant Rv0183; 2 , M. smegmatis mc ${ }^{2} 155 \mathrm{WT}$; 3 , M. smegmatis MSMEG $\Delta 0220$ mutant strain; 4, MSMEG 0220 strain complemented with corresponding gene (ComMSMEG 0220 ); 5, strain complemented with gene encoding catalytically inactive, MSMEG_0220S110A (ComMSMEG_0220S110A; S110 corresponds to the catalytic serine of MSMEG_0220); 6, recombinant MSMEG_0220 purified protein $(100 \mathrm{ng}$ ). (B) Comparison of growth of the above strains of $M$. smegmatis on $7 \mathrm{H} 9$ agar supplemented with monoolein (MO). Colony forming units (CFU) were counted after 3-5 days of incubation at $37^{\circ} \mathrm{C}$. (C-J) Comparison of cultures of above M. smegmatis strains after 5 days of incubation at $37^{\circ} \mathrm{C}$ on $7 \mathrm{H} 11$ solid medium: WT (C and G), MSMEG 0220 (D and H), ComMSMEG 0220 (E and I), and ComMSMEG $\Delta 0220 S 110 \mathrm{~A}$ (F and J). (C-F) Comparison of colony morphologies after 5 days of incubation at $37{ }^{\circ} \mathrm{C}$ on $7 \mathrm{H} 11$ solid medium. Top view of the colonies showing rough (C, E, and F) or smooth (D) morphologies. (G-J) Optical microscopy (magnification, $\times 63$ ) observation of $M$. smegmatis cells grown in Tween 80-free 7H9 broth. Cultures of MSMEG 0220 strain (H) were a homogeneous cell suspension in this medium, while those of the other strains showed bacterial aggregates (Adapted from Ref. [40]).

phospholipase A by specifically hydrolyzing phospholipids, Cfp21 exhibits a lipolytic activity on triolein and showed a preference for $\mathrm{C}_{8}$ fatty acyl chains monoester substrates [8]. In vitro cytotoxic assays on macrophages demonstrated that Cut4 induces macrophage lysis, presumably as a consequence of its phospholipase activity. These observations support the view that cutinases interact with the host membranes and participate in the virulence/ infection process by degrading host lipids. Another member of the cutinase family, Cut6 (Rv3802) and its homologs in M. smegmatis or Corynebacterium, have been found to be essential for in vitro viability [43,44], and the lipid analysis associated with their thioesterase activity suggested these enzymes may participate to mycolic acid biosynthesis [45]. Moreover, Cut6 was able to trigger a specific T-lymphocyte response and was able to protect mice from a challenge with $M$. tuberculosis, and hence is regarded as a potential vaccine antigen [36].

\section{Phospholipase C}

In infected macrophages, $M$. tuberculosis resides within a vacuole that exhibits characteristics of an early endosomal vesicle. It has been shown that mycobacterial phospholipids can mimic 
host lipids and inhibit full endocytic maturation of the pathogencontaining vacuole [46]. Phospholipase C, phospholipase D, and sphingomyelinase activities have been detected in $M$. tuberculosis [47]. M. tuberculosis phospholipases resemble those encoded by Pseudomonas aeruginosa $\mathrm{plcH}$ and $\mathrm{plcN}$ genes, which contribute to the virulence of this pathogen. The role of phospholipase in intracellular survival and as a virulence factor has been demonstrated in bacterial species such as Listeria monocytogenes [48]. M. tuberculosis genome carries three adjacently located phospholipase C (PLC) genes ( $p l c A, p l c B$ and $p l c C$ ) that are similar to the $P$. aeruginosa plc genes and a fourth truncated gene ( $p l c D)$, separated from this cluster [49]. Bakala N'goma et al. [5] were able to obtain high yields of all four pure recombinant $M$. tuberculosis PLCs and demonstrated that all four enzymes are cytotoxic to mouse macrophages owing to their hydrolytic activity on cell membrane phospholipids. Previous work from Raynaud et al. has shown that triple and quadruple M. tuberculosis plc-knock-out mutants were attenuated in the late phase of infection in a mouse model [50]. On the other hand, $M$. bovis, the causative agent of bovine TB, naturally lacks the plcABC gene cluster which is a part of the RD5 genomic deletion in this strain, and the remaining gene, plcD gene is inactivated by an IS6110 insertion [51]. PLC enzymes may contribute to TB infection through their contact-dependent cytolytic activity, possibly by lysing the phagolysosomal membrane [52]. However, several studies devoted to mycobacterial PLCs have failed to identify the exact function of these enzymes in pathogenesis.

\section{Lipolytic enzymes as new TB diagnostic biomarkers}

Several reports have highlighted the failure of conventional diagnostic tools in the precise detection of $\mathrm{TB}$, and this problem is more acute in the diagnosis of newly infected patients where the immune system may not be fully primed against antigens [53]. Some of the purified antigens that have been tested, showed highly variable sensitivity and specificity in serological tests for TB [54]. The localization of the mycobacterial lipolytic enzymes in the cell wall or at the cell surface, and their involvement in the host cell lipid degradation discussed above prompted investigators to hypothesize that these proteins may also participate in the modulation of the immune response $[35,36]$ and/or in the invasion of host cells $[5,8]$. These enzymes may therefore be considered as biomarkers of active TB.

The use of LipY as a serodiagnostic marker was first suggested in a study reporting the induction of a strong anti-LipY humoral response in TB patients living in an endemic area [35]. These initial observations were recently extended to LipY, Rv0183, Cfp21, and Cut4, and evaluated in European patients with either active TB or latent TB compared to non-infected population groups (Fig. 5A and 5B) [31]. Strong IgG-specific humoral responses were detected in TB patients against these antigens, compared to a low background in healthy volunteers and in BCG-vaccinated healthy individuals. Among these four markers, Cut4 presented the best performance with a specificity of $94.6 \%$ and a sensitivity of $90.5 \%$ in a study population including 149 healthy individuals and 105 active TB patients. In addition, $87 \%$ of smear-negative but culture-positive patients were positive for Cut4 serological test, indicating an excellent sensitivity of this assay. The Cut4-based ELISA also allowed discriminating patients with active TB from those with a latent infection. This may be related to higher expression levels of Cut 4 during the active phase of the disease when bacilli are actively replicating.

Immunoscreening of an expression library of $M$. tuberculosis genomic DNA showed that LipC was expressed during the early stages of active infection. Consistent with its localization at the bacterial surface, LipC was found to induce an antibody response in patients with active TB co-infected or not with HIV (Fig. 5C), which helped to distinguish TB patients from TB-uninfected individuals. Lastly, immunodominant epitopes of LipC that are exposed on outer surface of the protein were identified and proposed as ingredients for future peptide-based serodiagnostic tests [32]. M. tuberculosis possesses an additional cell wall-associated protein, LipF, exhibiting phospholipase $C$ activity. The recombinant enzyme was shown to hydrolyze phosphatidylcholine in vitro [55] and induce a significant humoral B cell response in children suffering from TB. Therefore, together with LipF, PLCs should be evaluated for their potential in TB diagnosis.

The above studies suggest that these strongly immunogenic lipolytic enzymes would allow distinguishing active from latent TB infections with high sensitivity and specificity. Further studies, including larger numbers of sera are now required to confirm these initial observations. This is particularly warranted for a better TB control, and in endemic countries, where application in simple immunoassays may allow rapid, sensitive, and inexpensive detection of cases missed by standard sputum smear microscopy.

\section{Summary and future prospects}

A key feature in the success of M. tuberculosis as a pathogen is its ability to evade host immunity and to establish a chronic and persistent infection. Based on the observation of an unusually large number of lipases/phospholipases in M. tuberculosis, and the studies on their biological properties, this family of enzymes appears to play a central role in the virulence and survival strategies employed by this bacterium to persist in the host. During the infection process, secreted mycobacterial lipolytic enzymes catalyze the hydrolysis of host cell lipids. The released fatty acids are then taken up by intracellular mycobacteria and stored in the form of TAGs to be used as sources of carbon and energy during the persistent stage. Conversely, intracellular TAG hydrolases may be required for assimilation of ILIs to exit dormancy. Thus, these enzymes seem to be involved throughout the life-cycle of the pathogen as catalytic and perhaps also as structural entities.

Lipolytic enzymes may hence be considered as potent therapeutic targets for developing future anti-TB drugs. Indeed, specific inhibitors that would interfere with the consumption of host cell lipids by the infecting mycobacteria may be able to prevent or shorten the persistent state. As an example, tetrahydrolipstatin, also known as Orlistat, an inhibitor of digestives lipases used to treat obesity, was found to significantly impact the growth of $M$. smegmatis [10] and exhibits potent anti-mycobacterial activity on several species, including $M$. tuberculosis [23]. Tetrahydrolipstatin is not the only candidate and other drugs are being tested right now in academic and industrial laboratory. However, undesirable effects may occur due to possible interactions between these inhibitors and human lipases, thus raising important safety issues that must be taken into consideration in such therapeutic approaches. Lipolytic enzymes could also be considered as potential vaccine candidates as demonstrated by West et al. [42]. Indeed, authors showed that cutinases delivered as DNA vaccines were able to induce a significant protection in a mouse model of $M$. tuberculosis infection.

In view of their important role in the pathogenesis of the disease, and the occurrence of seroreactivity in patients, lipolytic enzymes are being considered as new diagnostic markers. In particular, these markers allowed to override the background signals arising from prior BCG vaccination and appear as potent candidates to distinguish active TB from latent TB infections. These enzymes may be exploited for diagnosis of TB in smear-negative, HIV-infected individuals in countries highly afflicted by HIV and 
Version définitive du manuscrit publiée dans / Final version of the manuscript published in :

Biochimie (2013), Vol. 95, p. 66-73, DOI: 10.1016/j.biochi.2012.07.008

Journal homepage : www.elsevier.com/locate/biochi
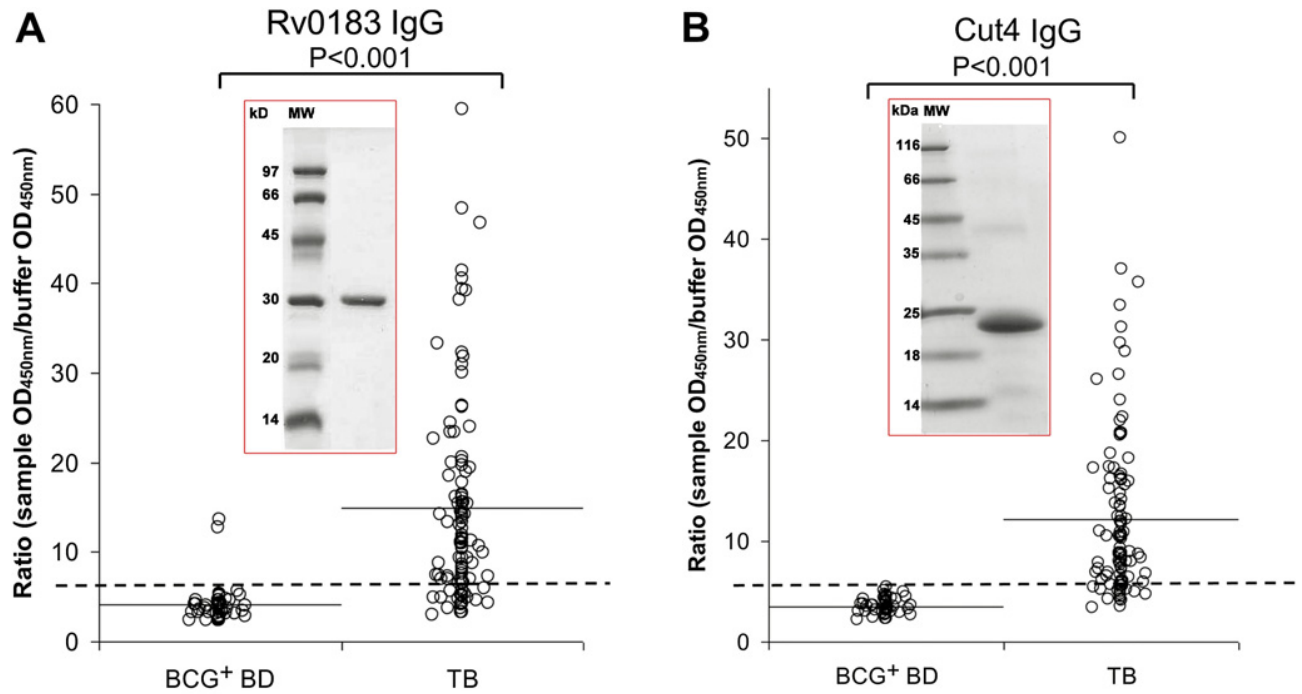

C

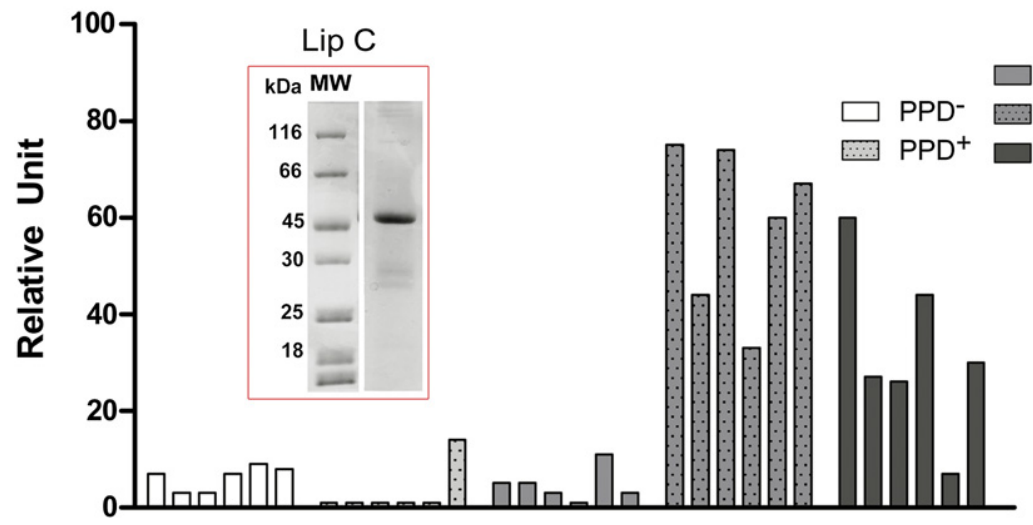

Fig. 5. Immunoserological response to recombinant lipolytic enzymes in TB patients and healthy individuals. (A and B), IgG humoral response in serum samples from TB patients or BCG-vaccinated healthy individuals tested against recombinant lipolytic enzymes, Rv0183 (A) or Cut4 (B). Horizontal continuous lines represent the mean values of the response, whereas dotted lines represent the cut-off values defined in the study. BCG ${ }^{+}$BD, BCG-vaccinated blood donors $(n=50)$, TB, active TB patients $(n=105)$. (C) Immunoreactivity of recombinant LipC with sera from TB patients $\left(\mathrm{HIV}^{+} \mathrm{TB}^{+}\right.$and $\mathrm{HIV}^{-} \mathrm{TB}^{+}$, each $n=6$ ) and control subjects $\left(\mathrm{PPD}^{-}, \mathrm{PPD}^{+}, \mathrm{HIV}^{+} \mathrm{TB}^{-}\right.$, each $n=6$ ). Relative immunoreactivity was calculated using the "Image J" software by determining the intensity of the recombinant LipC-6His band against anti-LipC compared to that against anti-His antibody that was considered as $100 \%$. The purity of each recombinant enzyme was verified by SDS-PAGE (Adapted from Refs. [31,32]).

TB infections. The potential of lipolytic enzymes in diagnosis should also be investigated in infants, as their immune responses to a TB infection can be different from that in adults.

Because these enzymes are shared by several mycobacterial species, they represent a huge field of exploration not only for TB but also for other mycobacterial diseases. These include, for instance, Buruli ulcer caused by Mycobacterium ulcerans and pulmonary infections caused by Mycobacterium abscessus, an emerging pathogen in patients with underlying lung disorders such as cystic fibrosis. $M$. tuberculosis expresses a plethora of lipolytic enzymes, of which only a limited number have been studied so far and much remains to be learned about the diversity of their biological functions. The mycobacterial lipolytic enzymes are thus a gold mine for research in understanding and combating TB.

\section{Acknowledgments}

This work and the post-doctoral fellowship to Luc Dedieu were funded by the Agence Nationale de la Recherche in the framework of the ANR-Foamy-TuB grant (ANR MIEN 2009-00904) to Stéphane Canaan and Laurent Kremer, by the LISA Carnot Institute (Convention ANR $n^{\circ}$ 07-CARN-009-01) and by the CNRS and the
Aix-Marseille Université. Authors thank Anuradha Alahari for help in editing the manuscript.

\section{References}

[1] WHO, Global Tuberculosis Control 2011, http://www.who.int/tb/publications/ global_report/en/index.html.

[2] D.E. Minnikin, L. Kremer, L.G. Dover, G.S. Besra, The methyl-branched fortifications of Mycobacterium tuberculosis, Chem. Biol. 9 (2002) 545-553.

[3] O. Neyrolles, C. Guilhot, Recent advances in deciphering the contribution of Mycobacterium tuberculosis lipids to pathogenesis, Tuberculosis (Edinb.) 91 (2011) 187-195.

[4] N. Garton, H. Christensen, D. Minnikin, R. Adegbola, M. Barer, Intracellular lipophilic inclusions of mycobacteria in vitro and in sputum, Microbiology 148 (2002) 2951-2958.

[5] J.C. Bakala N'goma, M. Schue, F. Carriere, A. Geerlof, S. Canaan, Evidence for the cytotoxic effects of Mycobacterium tuberculosis phospholipase $C$ towards macrophages, Biochim. Biophys. Acta 1801 (2010) 1305-1313.

[6] K. Côtes, R. Dhouib, I. Douchet, H. Chahinian, A. de Caro, F. Carriere, S. Canaan, Characterization of an exported monoglyceride lipase from Mycobacterium tuberculosis possibly involved in the metabolism of host cell membrane lipids, Biochem. J. 408 (2007) 417-427.

[7] O. Neyrolles, R. Hernandez-Pando, F. Pietri-Rouxel, P. Fornes, L. Tailleux J.A. Payan, E. Pivert, Y. Bordat, D. Aguilar, M.C. Prevost, C. Petit, B. Gicquel, Is adipose tissue a place for Mycobacterium tuberculosis persistence? PLoS ONE 1 (2006) e43.

[8] M. Schué, D. Maurin, R. Dhouib, J.C. N'Goma, V. Delorme, G. Lambeau, F. Carriere, S. Canaan, Two cutinase-like proteins secreted by Mycobacterium 
tuberculosis show very different lipolytic activities reflecting their physiological function, FASEB J. 24 (2010) 1893-1903.

[9] J. Daniel, H. Maamar, C. Deb, T.D. Sirakova, P.E. Kolattukudy, Mycobacterium tuberculosis uses host triacylglycerol to accumulate lipid droplets and acquires a dormancy-like phenotype in lipid-loaded macrophages, PLOS Pathog. 7 (2011) e1002093.

[10] R. Dhouib, A. Ducret, P. Hubert, F. Carriere, S. Dukan, S. Canaan, Watching intracellular lipolysis in mycobacteria using time lapse fluorescence microscopy, Biochim. Biophys. Acta 1811 (2011) 234-241.

[11] E.J. Munoz-Elias, J.D. McKinney, Mycobacterium tuberculosis isocitrate lyases 1 and 2 are jointly required for in vivo growth and virulence, Nat. Med. 11 (2005) 638-644.

[12] P. Peyron, J. Vaubourgeix, Y. Poquet, F. Levillain, C. Botanch, F. Bardou, M. Daffe, J.F. Emile, B. Marchou, P.J. Cardona, C. de Chastellier, F. Altare, Foamy macrophages from tuberculous patients' granulomas constitute a nutrientrich reservoir for M. tuberculosis persistence, PLoS Pathog. 4 (2008) e1000204.

[13] K.L. Low, P.S. Rao, G. Shui, A.K. Bendt, K. Pethe T. Dick, M.R. Wenk, Triacylglycerol utilization is required for regrowth of in vitro hypoxic nonreplicating Mycobacterium bovis bacillus Calmette-Guerin, J. Bacteriol. 191 (2009) 5037-5043.

[14] M. Wältermann, A. Steinbüchel, Neutral lipid bodies in prokaryotes: recent insights into structure, formation, and relationship to eukaryotic lipid depots, J. Bacteriol. 187 (2005) 3607-3619.

[15] J. Daniel, C. Deb, V.S. Dubey, T.D. Sirakova, B. Abomoelak, H.R. Morbidoni, P.E. Kolattukudy, Induction of a novel class of diacylglycerol acyltransferases and triacylglycerol accumulation in Mycobacterium tuberculosis as it goes into a dormancy-like state in culture, J. Bacteriol. 186 (2004) 5017-5030.

[16] C. Deb, J. Daniel, T.D. Sirakova, B. Abomoelak, V.S. Dubey, P.E. Kolattukudy, A novel lipase belonging to the hormone-sensitive lipase family induced under starvation to utilize stored triacylglycerol in Mycobacterium tuberculosis, J. Biol. Chem. 281 (2006) 3866-3875.

[17] J.D. McKinney, K. Honer zu Bentrup, E.J. Munoz-Elias, A. Miczak, B. Chen, W.T. Chan, D. Swenson, J.C. Sacchettini, W.R. Jacobs Jr., D.G. Russell, Persistence of Mycobacterium tuberculosis in macrophages and mice requires the glyoxylate shunt enzyme isocitrate lyase, Nature 406 (2000) 735-738.

[18] K.L. Low, G. Shui, K. Natter, W.K. Yeo, S.D. Kohlwein, T. Dick, S.P. Rao, M.R. Wenk, Lipid droplet-associated proteins are involved in the biosynthesis and hydrolysis of triacylglycerol in Mycobacterium bovis bacillus Calmette-Guerin, J. Biol. Chem. 285 (2010) 21662-21670.

[19] K.A. Mattos, H. D’Avila, L.S. Rodrigues, V.G. Oliveira, E.N. Sarno, G.C. Atella, G.M. Pereira, P.T. Bozza, M.C. Pessolani, Lipid droplet formation in leprosy: toll-like receptor-regulated organelles involved in eicosanoid formation and Mycobacterium leprae pathogenesis, J. Leukoc. Biol. 87 (2010) 371-384.

[20] P.R. Wheeler, C. Ratledge, Use of carbon sources for lipid biosynthesis in Mycobacterium leprae: a comparison with other pathogenic mycobacteria, J. Gen. Microbiol. 134 (1988) 2111-2121.

[21] N.M. Parrish, J.D. Dick, W.R. Bishai, Mechanisms of latency in Mycobacterium tuberculosis, Trends Microbiol. 6 (1998) 107-112.

[22] K.A. Mattos, F.A. Lara, V.G. Oliveira, L.S. Rodrigues, H. D’Avila, R.C. Melo, P.P. Manso, E.N. Sarno, P.T. Bozza, M.C. Pessolani, Modulation of lipid droplets by Mycobacterium leprae in Schwann cells: a putative mechanism for host lipid acquisition and bacterial survival in phagosomes, Cell Microbiol. 13 (2011) 259-273.

[23] L. Kremer, C. de Chastellier, G. Dobson, K.J. Gibson, P. Bifani, S. Balor, J.P. Gorvel, C. Locht, D.E. Minnikin, G.S. Besra, Identification and structural characterization of an unusual mycobacterial monomeromycolyl-diacylglycerol, Mol. Microbiol. 57 (2005) 1113-1126.

[24] S. Talati, P.R. Mahadevan, Lipase activity in Mycobacterium leprae-an indicator of metabolic function, Indian J. Lepr. 58 (1986) 367-372.

[25] J.E. Hawkins, W. Steenken, Lipase activity of mycobacteria, Am. Rev. Respir. Dis. 87 (1963) 585-588

[26] A. Andrejew, J. Desbordes, Hydrolysis of Fatty Acids Esters by Mycobacterium phlei, vol. 117, Ann Inst Pasteur, Paris, 1969, pp. 486-500.

[27] G. Singh, D. Jadeja, J. Kaur, Lipid hydrolizing enzymes in virulence: Mycobacterium tuberculosis as a model system, Crit. Rev. Microbiol. 36 (2010) 259-269.

[28] A. Aloulou, F. Carriere, Gastric lipase: an extremophilic interfacial enzyme with medical applications, Cell Mol. Life Sci. 65 (2008) 851-854.

[29] B. Fielding, Tracing the fate of dietary fatty acids: metabolic studies of postprandial lipaemia in human subjects, Proc. Nutr. Soc. 70 (2011) 342-350.

[30] A.D. Quiroga, R. Lehner, Liver triacylglycerol lipases, Biochim. Biophys. Acta (2011).

31] B. Brust, M. Lecoufle, E. Tuaillon, L. Dedieu, S. Canaan, V. Valverde, L. Kremer, Mycobacterium tuberculosis lipolytic enzymes as potential biomarkers for the diagnosis of active tuberculosis, PLoS ONE 6 (2011) e25078.

[32] G. Shen, K. Singh, D. Chandra, C. Serveau-Avesque, D. Maurin, S. Canaan, R. Singla, D. Behera, S. Laal, LipC (Rv0220) is an immunogenic cell surface esterase of Mycobacterium tuberculosis, Infect. Immun. 80 (2012) 243-253.

[33] S.T. Cole, R. Brosch, J. Parkhill, T. Garnier, C. Churcher, D. Harris, S.V. Gordon, K. Eiglmeier, S. Gas, C.E. Barry 3rd, F. Tekaia, K. Badcock, D. Basham, D. Brown, T. Chillingworth, R Connor, R. Davies, K. Devlin, T. Feltwell, S. Gentles, N. Hamlin, S. Holroyd, T. Hornsby, K. Jagels, B.G. Barrell, et al., Deciphering the biology of Mycobacterium tuberculosis from the complete genome sequence, Nature 393 (1998) 537-544.

[34] D.L. Ollis, E. Cheah, M. Cygler, B. Dijkstra, F. Frolow, S.M. Franken, M. Harel, S.J. Remington, I. Silman, J. Schrag, J.L. Sussman, K.H.G. Verschueren, A. Goldman, The $\alpha / \beta$ hydrolase fold, Protein Eng. 5 (1992) 197-211.

[35] K.C. Mishra, C. de Chastellier, Y. Narayana, P. Bifani, A.K. Brown, G.S. Besra, V.M. Katoch, B. Joshi, K.N. Balaji, L. Kremer, Functional role of the PE domain and immunogenicity of the Mycobacterium tuberculosis triacylglycerol hydrolase LipY, Infect. Immun. 76 (2008) 127-140.

[36] E.R. Shanahan, R. Pinto, J.A. Triccas, W.J. Britton, N.P. West, Cutinase-like protein- 6 of Mycobacterium tuberculosis is recognised in tuberculosis patients and protects mice against pulmonary infection as a single and fusion protein vaccine, Vaccine 28 (2010) 1341-1346.

[37] M.H. Daleke, A. Cascioferro, K. de Punder, R. Ummels, A.M. Abdallah, N. van der Wel, P.J. Peters, J. Luirink, R. Manganelli, W. Bitter, Conserved Pro-Glu (PE) and Pro-Pro-Glu (PPE) protein domains target LipY lipases of pathogenic mycobacteria to the cell surface via the ESX-5 pathway, J. Biol. Chem. 286 (2011) 19024-19034.

[38] D. Bottai, M. Di Luca, L. Majlessi, W. Frigui, R. Simeone, F. Sayes, W. Bitter, M.J. Brennan, C. Leclerc, G. Batoni, M. Campa, R. Brosch, S. Esin, Disruption of the ESX-5 system of Mycobacterium tuberculosis causes loss of PPE protein secretion, reduction of cell wall integrity and strong attenuation, Mol. Microbiol. (2012).

[39] J. Zeng, L. Zhang, Y. Li, Y. Wang, M. Wang, X. Duan, Z.G. He, Over-producing soluble protein complex and validating protein-protein interaction through a new bacterial co-expression system, Protein Expr. Purif. 69 (2010) 47-53.

[40] R. Dhouib, F. Laval, F. Carriere, M. Daffe, S. Canaan, A monoacylglycerol lipase from Mycobacterium smegmatis involved in bacterial cell interaction, J. Bacteriol. 192 (2010) 4776-4785.

[41] G. Xu, H. Jia, Y. Li, X. Liu, M. Li, Y. Wang, Hemolytic phospholipase Rv0183 of Mycobacterium tuberculosis induces inflammatory response and apoptosis in alveolar macrophage RAW264.7 cells, Can. J. Microbiol. 56 (2010) 916-924.

[42] N.P. West, F.M. Chow, E.J. Randall, J. Wu, J. Chen, J.M. Ribeiro, W.J. Britton, Cutinase-like proteins of Mycobacterium tuberculosis: characterization of their variable enzymatic functions and active site identification, FASEB J. 23 (2009) 1694-1704.

[43] P.K. Crellin, J.P. Vivian, J. Scoble, F.M. Chow, N.P. West, R. Brammananth, N.I. Proellocks, A. Shahine, J. Le Nours, M.C. Wilce, W.J. Britton, R.L. Coppel, J. Rossjohn, T. Beddoe, Tetrahydrolipstatin inhibition, functional analyses, and three-dimensional structure of a lipase essential for mycobacterial viability, J. Biol. Chem. 285 (2010) 30050-30060.

[44] X. Meniche, C. Labarre, C. de Sousa-d'Auria, E. Huc, F. Laval, M. Tropis, N. Bayan, D. Portevin, C. Guilhot, M. Daffe, C. Houssin, Identification of a stress-induced factor of Corynebacterineae that is involved in the regulation of the outer membrane lipid composition, J. Bacteriol. 191 (2009) 7323-7332.

[45] S.K. Parker, R.M. Barkley, J.G. Rino, M.L. Vasil, Mycobacterium tuberculosis Rv3802c encodes a phospholipase/thioesterase and is inhibited by the antimycobacterial agent tetrahydrolipstatin, PLoS ONE 4 (2009) e4281.

[46] R.A. Fratti, J. Chua, I. Vergne, V. Deretic, Mycobacterium tuberculosis glycosylated phosphatidylinositol causes phagosome maturation arrest, Proc. Natl. Acad. Sci. U. S. A. 100 (2003) 5437-5442.

[47] K. Johansen, R. Gill, M. Vasil, Biochemical and molecular analysis of phospholipase $C$ and phospholipase D activity in mycobacteria, Infect. Immun. 64 (1996) 3259-3266

[48] D.H. Schmiel, V.L. Miller, Bacterial phospholipases and pathogenesis, Microbes Infect. 1 (1999) 1103-1112.

[49] T. Matsui, C.R. Carneiro, S.C. Leao, Evidence for the expression of native Mycobacterium tuberculosis phospholipase C: recognition by immune sera and detection of promoter activity, Braz. J. Med. Biol. Res. 33 (2000) 1275-1282.

[50] C. Raynaud, C. Guilhot, J. Rauzier, Y. Bordat, V. Pelicic, R. Manganelli, I. Smith, B. Gicquel, M. Jackson, Phospholipases $C$ are involved in the virulence of Mycobacterium tuberculosis, Mol. Microbiol. 45 (2002) 203-217.

[51] S.V. Gordon, R. Brosch, A. Billault, T. Garnier, K. Eiglmeier, S.T. Cole, Identification of variable regions in the genomes of tubercle bacilli using bacterial artificial chromosome arrays, Mol. Microbiol. 32 (1999) 643-655.

[52] C.H. King, S. Mundayoor, J.T. Crawford, T.M. Shinnick, Expression of contactdependent cytolytic activity by Mycobacterium tuberculosis and isolation of the genomic locus that encodes the activity, Infect. Immun. 61 (1993) $2708-2712$.

[53] S. Kunnath-Velayudhan, M.L. Gennaro, Immunodiagnosis of tuberculosis: a dynamic view of biomarker discovery, Clin. Microbiol. Rev. 24 (2011) $792-805$.

[54] K.R. Steingart, N. Dendukuri, M. Henry, I. Schiller, P. Nahid, P.C. Hopewell, A. Ramsay, M. Pai, S. Laal, Performance of purified antigens for serodiagnosis of pulmonary tuberculosis: a meta-analysis, Clin. Vaccine Immunol. 16 (2009) 260-276

[55] M. Srinivas, S. Rajakumari, Y. Narayana, B. Joshi, V.M. Katoch, R. Rajasekharan, K.N. Balaji, Functional characterization of the phospholipase $\mathrm{C}$ activity of Rv3487c and its localization on the cell wall of Mycobacterium tuberculosis, J. Biosci. 33 (2008) 221-230. 